mental material than animal cells. Illustrations of this were provided by discussions about the difficulty of finding out which of the cell surface nucleosidases are associated with ion transport. Nevertheless Professor A. Kylin (University of Stockholm) presented evidence that ATPase stimulated by $\mathrm{Na}^{+}$and $\mathrm{K}^{+}$ could be isolated from a variety of plant tissue.

Plant cells usually contain a large central vacuole, necessary for maintaining a rigid cell wall, which has often made accurate analyses of internal ion concentrations difficult. Dr A. Lauchli (Technische Hochschule, Darmstadt) showed, however, that electron probe analysis and other physical techniques offer hope that reliable values may soon be obtained.

Progress in these areas is bound to be slow; but substantial progress has been made in the elucidation of active ion movements. A joint paper by Dr J. Raven (University of Dundee) and Dr F. A. Smith (University of Adelaide) crystallized the ideas of many, and now it seems likely that the outer membranes of plant cells will have to be thought of as helping to regulate the cytoplasmic $p \mathrm{H}$ by pumping out $\mathrm{H}^{+}$. The proton pump seems to be electrogenic and Dr R. Spanswick (Cornell University) presented data for Nitella translucens to show that an understanding of the electrical properties of this pump can explain previously inexplicable information about piasmalemma conductance. Further work in this area is likely to be stimulated by the detailed analysis by Dr W. J. Vredenberg (Centre for Plant Physiological Research, Wageningen) of current-voltage characteristics of the plasmalemma of $N$. transiucens and by his suggestions concerning the relationship of the capacity of the proton pump and the changes in the energy state of the cell.

The net uptake of $\mathrm{Cl}^{-}$by plant and algal cells could well be associated with the $\mathrm{pH}$ regulating pump, but as yet there is no generally accepted mechanism. There is, however, according to Drs A. F. Hill and B. S. Hill (University of Cambridge), evidence that in the salt glands of Limonium active pumping of $\mathrm{Cl}^{-}$is associated with ATPase activity.

The control of ion transport in higher plants seems set to be one of the next areas of intensive research. Dr M. G. Pitman and J. Cram (University of Sydney) set the scene with their survey of the problem. Abscisic acid is likely to be an important messenger for the control processes and Dr T. Collins (University of Liverpool) provided support for this from a comparison of the in vitro effect of the hormone and induced in vivo changes of ion content and root exudation. Future studies will be heavily dependent on information about in vivo hormone levels.
PARASITOLOGY

\section{Use of Genetic Markers}

from a Correspondent

WHEN parentage is in doubt (as in the offspring of multiple matings), it can often be established by the use of genetic characters as markers. A novel use of this technique to ascertain the parentage of offspring from multiple infections of hosts by hymenopterous (wasp) parasites is reported by Holmes (Entomophaga, 17, $79 ; 1972$ ). Such multiple infection, in the absence of mechanisms which prevent it, occurs chiefly when the proportion of hosts attacked and the probability of local host extinction are close to their maximum values. The consequences of multiple infection are thus of crucial importance to the stability of the host-parasite system in terms of population dynamics and must be incorporated in any realistic model of this system.

It is known that female Nasonia vitripennis (Chalcidae) lay fewer eggs in hosts (housefly puparia) which have been parasitized previously, and that the proportion of male offspring increases in cases of multiple attack. Hymenoptera can control the sex ratio of their progeny ; fertilized eggs become diploid females and unfertilized eggs produce haploid males. The sex ratio change observed in $N$. vitripennis, however, could have been the result simply of better competitive ability of male parasite larvae in conditions of food shortage.

Holmes used three pure parasite strains differing in eye colour, and exposed each host to a known sequence of single, isolated parasites. The duration of exposure was short enough to keep the number of offspring below the maximum which could be supported by the host, thus rendering parasite deaths from competitive effects unlikely. The progeny of parasites which had attacked first, second and third could be identified from their eye colours. Holmes found an increase in the proportion of males resulting from each successive attack, as well as the expected decrease in number of offspring.

It is implicit in these findings that female $N$. vitripennis are able to discriminate, not merely between healthy and parasitized hosts, but between those which have been attacked once and those which have been attacked twice. The number of eggs laid and the proportion fertilized are varied accordingly. Experiments with this and other wasps have shown that a dramatic rise in the proportion of male progeny results when searching female parasites are allowed to interact with each other. Thus, in $N$. vitripennis there are at least two separate mechanisms which tend to increase the male/female ratio when there are high densities of parasites. This conforms with theoretical predictions. Parasites emerging from a single host usually mate with each other. In cases of single attack this amounts to complete inbreeding, and the optimum number of males is the minimum number necessary to fertilize all the females. Any additional males would be produced in lieu of females, thus reducing the total number of eggs laid. These additional males could only contribute to the spread of their parental genes by mating with females other than their sisters. Where previous parasitism is detected, the probability of such matings increases and, in consequence, so does the optimum proportion of males.

\title{
New Media for Affinity Chromatography
}

IN next Wednesday's Nature New Biology (August 30), Porath and Sundberg describe a new chemical scheme for the coupling of biological molecules to a column matrix. The use of this kind of procedure has become indispensable for the isolation from complex mixtures of substances of specific affinity for an available ligand. One of the limitations has been the difficulty of attaching a sufficient density of the ligand species to the matrix to achieve good yields, and there has thus been a search for new methods of activating the matrix material.

Porath and Sundberg show that the introduction of reactive hydroxylic groups offers many advantages. They used the phenolic hydroxyls of the polyhydric phenol, phloroglucinol, which is coupled to agarose beads by cross-linking with epichlorohydrin. The phenolic groups may then be activated to receive the relevant ligand by way of its amino groups in any of the usual ways, for example with cyanogen bromide. Porath and Sundberg, however, offer a new suggestion, namely divinylsulphone. This chemisorbent is rapid and efficient, and in the system described led to an increased capacity over the cyanogen bromide-treated matrix by a factor of 2.5. The agarose beads become stiffer after this treatment and have good flow properties in columns.

The system also offers the advantage that the link joining the chemisorbent to the column matrix is quite long, which diminishes the likelibood of steric impedance of the binding sites. As a demonstration of their method Porath and Sundberg coupled soyabean trypsin inhibitor to agarose and used a $1.4 \times$ $3.1 \mathrm{~cm}$ column of this material to catch trypsin. No less than $80 \mathrm{mg}$ was recovered from a single experiment. 